\title{
The revised distance of supernova remnant G15.4+0.1
}

\author{
H. Su ${ }^{1,2,3}$, M. F. Zhang ${ }^{1,2}$, H. Zhu ${ }^{1}$, and D. $\mathrm{Wu}^{1,4}$ \\ 1 Key Laboratory of Optical Astronomy, National Astronomical Observatories, Chinese Academy of \\ Sciences, Beijing 100012,China; hq_su@bao.ac.cn,zhuhui@bao.ac.cn \\ 2 University of Chinese Academy of Science, 19A Yuquan Road, Beijing 100049, China \\ 3 International Centre for Radio Astronomy Research, Curtin University, Bentley, WA 6102, Australia \\ 4 College of Information Science \& Technology, Beijing Normal University, Beijing 100875, China
}

\begin{abstract}
We measure the distance to the supernova remnant G15.4+0.1 which is likely associated with TeV source HESS J1818-154. We build the neutral hydrogen (HI) absorption and ${ }^{13} \mathrm{CO}$ spectra to supernova remnant G15.4+0.1 by employing the data from the Southern Galactic Plane Survey (SGPS) and the Hi/OH/Recombination line survey (THOR). The maximum absorption velocity of about $140 \mathrm{~km} \mathrm{~s}^{-1}$ constrains its lower limit distance of about $8.0 \mathrm{kpc}$. Further, the fact that the Hi emission feature at about $95 \mathrm{~km} \mathrm{~s}^{-1}$ seems no corresponding absorption suggests that G15.4+0.1 has likely an upper limit distance of about $10.5 \mathrm{kpc}$. ${ }^{13} \mathrm{CO}$ spectrum to the remnant supports our measurement. The new distance revises parameters on its associated pulsar wind nebula and $\mathrm{TeV}$ source.
\end{abstract}

Key words: ISM: supernova remnants - methods: data analysis - stars: distances

\section{INTRODUCTION}

The supernova remnant (SNR) G15.4+0.2, also called G15.42+0.18 (G15 hereafter), has a break-out morphology toward its south and a northern shell with a radius of about $6^{\prime}$ in $1420 \mathrm{MHz}$. The spectral index of G15 is $-0.62 \pm 0.03$ from 330 to $4800 \mathrm{MHz}$ (Supan et al., 2015; Sun et al., 2011) with its peak flux density of $5.6 \mathrm{Jy}$ at $1 \mathrm{GHz}$. G15 has a morphological correspondence with the TeV $\gamma$-ray source HESS J1818154 (Adrián-Martínez et al., 2011). The high energy radiation possibly originates from a pulsar wind nebula (PWN) although the pulsar has not been detected. Multiwavelength studies have been performed to explore their properties (e.g. Abramowski et al. 2014; Castelletti et al. 2013)

Distance is a key to further study this TeV SNR. A near distance for the remnant G15 was suggested by Castelletti et al. (2013) by analyzing a noisy HI absorption spectrum to the remnant. We make a further effort to obtain its reliable distance by taking advantage of new-released $\mathrm{HI} / \mathrm{OH} / \mathrm{Recombination} \mathrm{line} \mathrm{survey}$ (THOR, Beuther et al. 2016), the archive Southern Galactic Plane Survey (SGPS, McClure-Griffiths et al. 2005), the Very Large Array Galactic Plane Survey (VGPS, Stil et al. 2006), and the Galactic Ring Survey (GRS, Jackson et al. 2006) data. 
The SGPS has a resolution of $100^{\prime \prime}$ and a sensitivity of less than $1 \mathrm{mJy}^{\mathrm{beam}}{ }^{-1}$ and were observed by The Australia Telescope Compact Array (ATCA) and Parkes telescopes. The SGPS survey has a reliable absolute flux scale by including the Parkes data. The continuum data of SGPS near G15 region is currently not available. The THOR survey observed $\mathrm{HI}$, four $\mathrm{OH}, 19 \mathrm{H}_{\alpha}$ recombination lines, and the L-band continuum covering the northern Galactic plane $\left(15<l<67^{\circ}\right.$ and $\left.|b|<1^{\circ}\right)$. The THOR data has an angular resolution about $20^{\prime \prime}$. We convert the unit of $\mathrm{Jy} \mathrm{beam}^{-1}$ in the THOR data to Kelvin (K) using factors of 1536.20 for the cube data. The absolute scale of the surface brightness in the THOR data is not reliable due to lacking zero baseline. However, the relative scale is good enough for us to do Hi absorption analysis. In addition, we adopt the ${ }^{13} \mathrm{CO}$ data from the GRS to confirm the reality of the absorption peaks, because ${ }^{13} \mathrm{CO}$ is a tracer of cold and dense $\mathrm{H}_{2}$ molecular cloud.

\section{SPECTRA ANALYSIS}

We use well-tested methods in order to build a reliable HI absorption spectrum to G15 as possible (Tian et al., 2007; Tian \& Leahy, 2011; Zhu et al., 2013).

We improve the absorption spectrum extraction by two steps. We choose a background region which is an annulus around the source region. Our background is adjacent to the source region, which makes that the background spectrum in the source direction. We remove six point-like sources from both the continuum map and the data cube to avoid contamination (see Fig. 1). We show the Hi emission, the HI absorption, and the ${ }^{13} \mathrm{CO}$ emission spectra of G15 (see Fig. 2 and 3). We extract the HI emission spectra from our source and background regions and then calculate the difference between the two spectra. This difference spectrum is actually proportional to the absorption spectrum, which is used to do absorption analysis. We extract the ${ }^{13} \mathrm{CO}$ spectrum in our selected background region from the GRS data and compare with our absorption spectrum.

The maximum velocity $\mathrm{HI}$ absorption feature appears at about $140 \mathrm{~km} \mathrm{~s}^{-1}$ (see Fig. 2] and 3). The ${ }^{13} \mathrm{CO}$ has data between $-5 \mathrm{~km} \mathrm{~s}^{-1}$ and $135 \mathrm{~km} \mathrm{~s}^{-1}$. There is, in fact, a ${ }^{13} \mathrm{CO}$ emission feature at $135 \mathrm{~km} \mathrm{~s}^{-1}$ which seems corresponding with the HI maximum velocity absorption feature. The rms of $0.009 \mathrm{~K}$ is obtained by averaging no ${ }^{13} \mathrm{CO}$ emission-line velocity ranges from -5 to $10 \mathrm{~km} \mathrm{~s}^{-1}$ and from 60 to $120 \mathrm{~km} \mathrm{~s}^{-1}$. The ${ }^{13} \mathrm{CO}$ peak temperature at $135 \mathrm{~km} \mathrm{~s}^{-1}$ is $0.047 \mathrm{~K}$, which is a $5 \sigma$ detection. This supports the HI absorption feature at about $140 \mathrm{~km} \mathrm{~s}^{-1}$ is real. The velocity of $140 \mathrm{~km} \mathrm{~s}^{-1}$ corresponds to a distance of about $8 \mathrm{kpc}$ based on the Galactic rotation model with $\Theta_{\odot}=220 \mathrm{~km} \mathrm{~s}^{-1}$ and $\mathrm{R}_{\odot}=8.5 \mathrm{kpc}$ (Fich et al., 1989). Thus, the lower limit distance of G15 is $8 \mathrm{kpc}$.

The Hi emission at a velocity of about $95 \mathrm{~km} \mathrm{~s}^{-1}$ seems no responsible absorption, which means G15 is likely located in front of this HI cloud. This velocity corresponds to a far side distance of about $10.5 \mathrm{kpc}$. The absorption peak at the velocity of about $-20 \mathrm{~km} \mathrm{~s}^{-1}$ is possibly caused by HI emission fluctuation so likely not a real feature (see the Hi emission spectra in Figs. 2] and 3). Similar fake absorption feature is often seen in faint SNRs (e.g. Leahy \& Tian 2008).

\section{RESULTS AND BRIEF DISCUSSION}

We obtain a new distance of $9.3 \pm 1.3 \mathrm{kpc}$ for G15. This is consistent with the $10 \pm 3 \mathrm{kpc}$ derived from the surface brightness vs. diameter relation of Galactic SNRs (Adrián-Martínez et al., 2011). Castelletti et al. 
(2013) showed an Hi absorption spectrum and suggested a smaller distance for the remnant. However, we do not find a clue from their paper to reproduce their spectrum, so we believe our careful measurement is more reliable.

The HI shell of G15 is $16.5 \mathrm{pc}$ in radius (for 6.1 arcmin) at a distance of $9.3 \mathrm{kpc}$. The PWN inside G15 has a total energy of less than $1.2 \times 10^{50}$ erg scaled from the value derived in Abramowski et al. (2014).

We revise the parameters of G15 using the shell-type model of SNR in Sturner et al. (1997). We assume the progenitor supernova of G15 has an explosion energy of $10^{51} \mathrm{erg}$, a mass of $8 \mathrm{M}_{\odot}$, a magnetic field strength of $1 \mu \mathrm{G}$, and an interstellar medium (ISM) density of 7 hydrogen atoms per cube centimeter, so we get the expansion velocity of about $700 \mathrm{~km} \mathrm{~s}^{-1}$, an age of $11 \mathrm{kyr}$, and a total atomic gas mass that forms the G15 shell of $3.3 \times 10^{3} \mathrm{M}_{\odot}$. The estimated parameters may have a 50 percent uncertainty due to the large variance of the interstellar medium density.

\section{ACKNOWLEDGEMENTS}

We thank Dr. Wenwu Tian for his initial questioning the SNR G15.4+0.1's distance issue and helpful comments during our writing the paper. We acknowledge support from the NSFC (11473038, 11273025). This publication makes use of molecular line data from the Boston University-FCRAO Galactic Ring Survey (GRS).

\section{References}

Abramowski, A., Aharonian, F., Ait Benkhali, F., et al. 2014, A\&A, 562, A40

Adrián-Martínez, S., Aguilar, J. A., Samarai, I. A., et al. 2011, arXiv:1112.0478

Beuther, H., Bihr, S., Rugel, M., et al. 2016, A\&A, 595, A32

Castelletti, G., Supan, L., Dubner, G., Joshi, B. C., \& Surnis, M. P. 2013, A\&A, 557, L15

Fich, M., Blitz, L., \& Stark, A. A. 1989, ApJ, 342, 272

Jackson, J. M., Rathborne, J. M., Shah, R. Y., et al. 2006, ApJS, 163, 145

Leahy, D. A., \& Tian, W. W. 2008, A\&A, 480, L25

McClure-Griffiths, N. M., Dickey, J. M., Gaensler, B. M., et al. 2005, ApJS, 158, 178

Stil, J. M., Taylor, A. R., Dickey, J. M., et al. 2006, AJ, 132, 1158

Sturner, S. J., Skibo, J. G., Dermer, C. D., \& Mattox, J. R. 1997, ApJ, 490, 619

Sun, X. H., Reich, P., Reich, W., et al. 2011, A\&A, 536, A83

Supan, L., Castelletti, G., Joshi, B. C., Surnis, M. P., \& Supanitsky, D. 2015, A\&A, 576, A81

Tian, W. W., \& Leahy, D. A. 2011, ApJ, 729, L15

Tian, W. W., Leahy, D. A., \& Wang, Q. D. 2007, A\&A, 474, 541

Zhu, H., Tian, W. W., Torres, D. F., Pedaletti, G., \& Su, H. Q. 2013, ApJ, 775, 95 


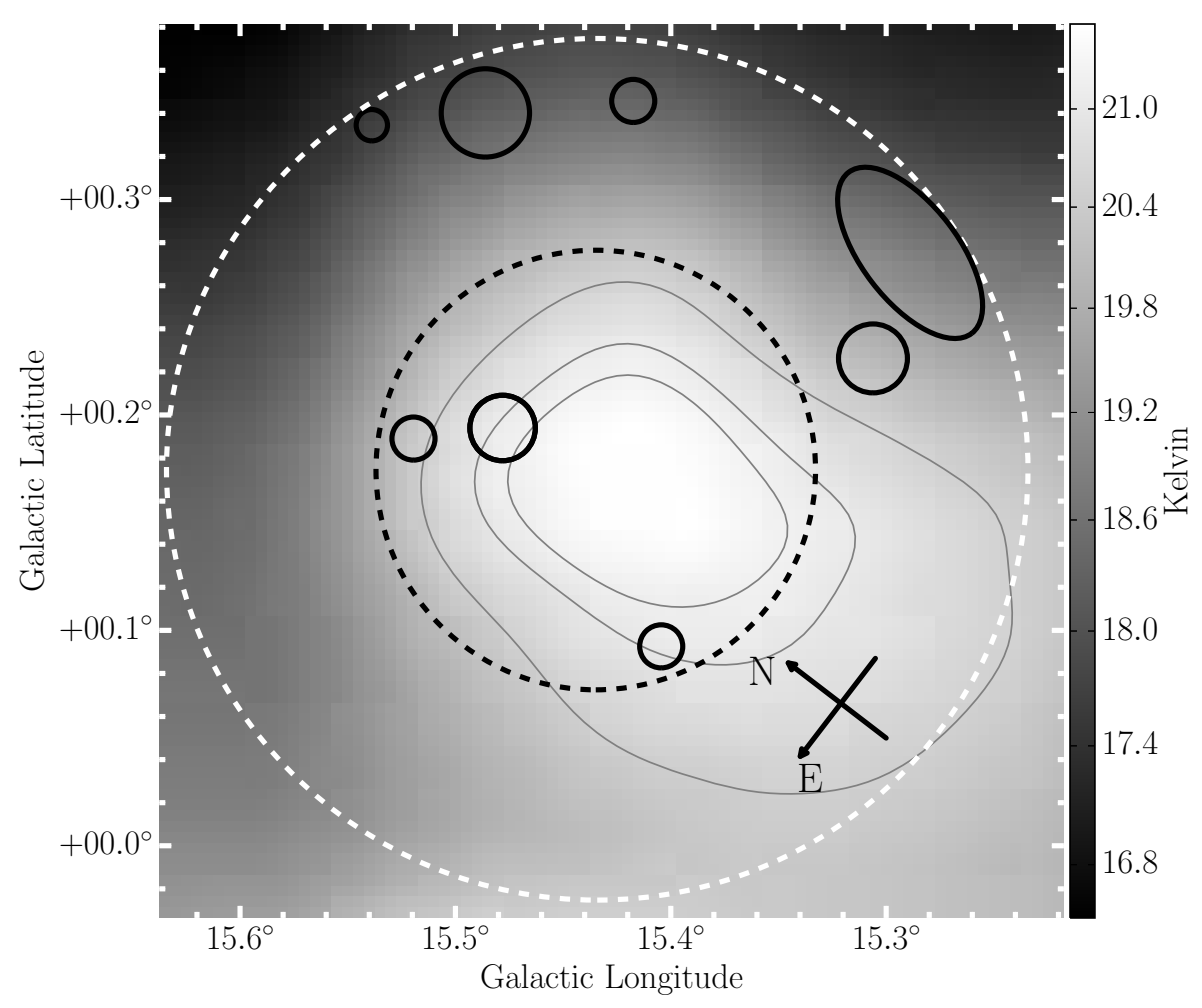

Fig. 1 The image of G15 at $1420 \mathrm{MHz}$ from the VGPS data. The black dashed circle shows the source region (' $\mathrm{ON}$ ' direction) with its center at $(\mathrm{g}, \mathrm{l})=\left(15.43^{\circ}, 0.17^{\circ}\right)$ and a radius of 6 ' 1 . The region between the white solid circle and the white dashed circle is the background region ('OFF' direction). The black circles show point-like sources from the THOR continuum image, which are excluded from our analysis. The black solid ellipse shows a bright source in the SGPS HI channel maps, which is also flagged in our spectra analysis. The gray contour levels are 20.5, 21.0, and 21.2 Kelvin. 


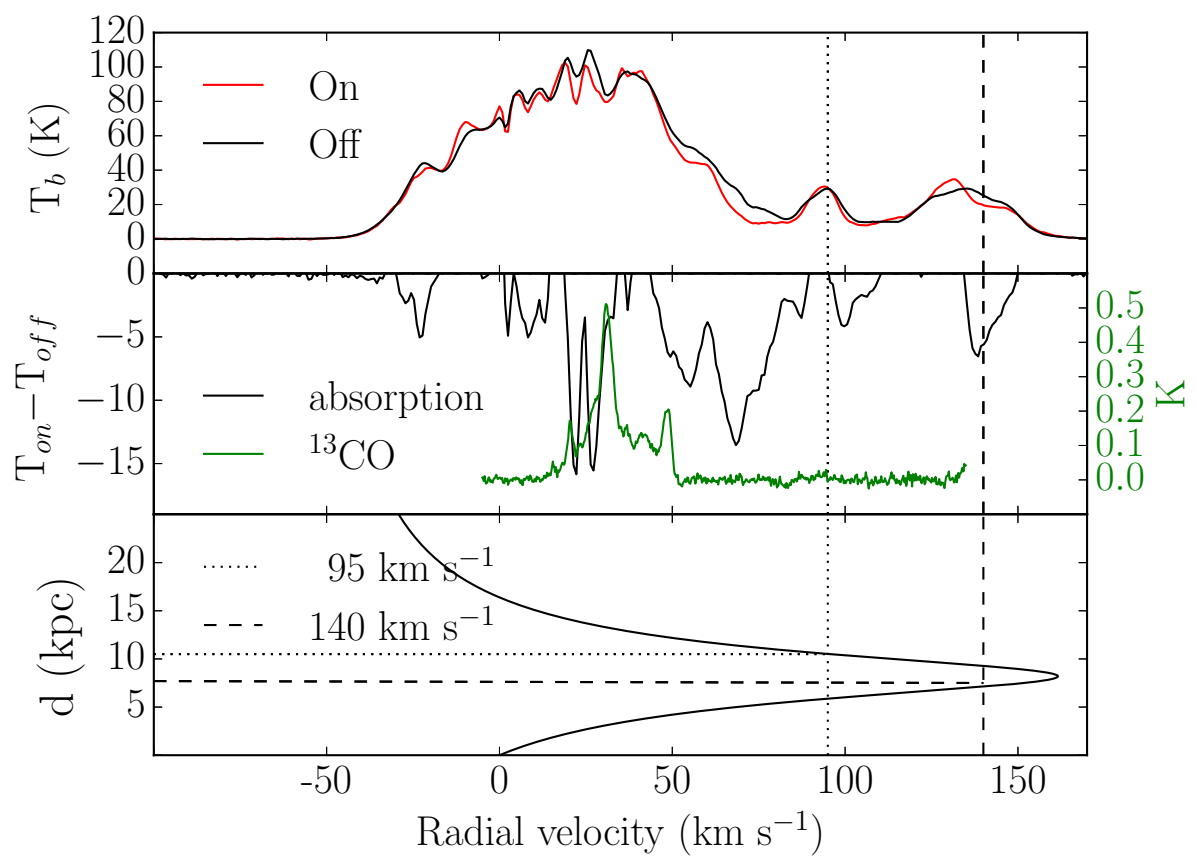

Fig. 2 The HI emission, HI absorption, and the ${ }^{13} \mathrm{CO}$ spectra of G15 from SGPS and GRS data. The bottom panel shows the relationship between the radial velocity and the distance.

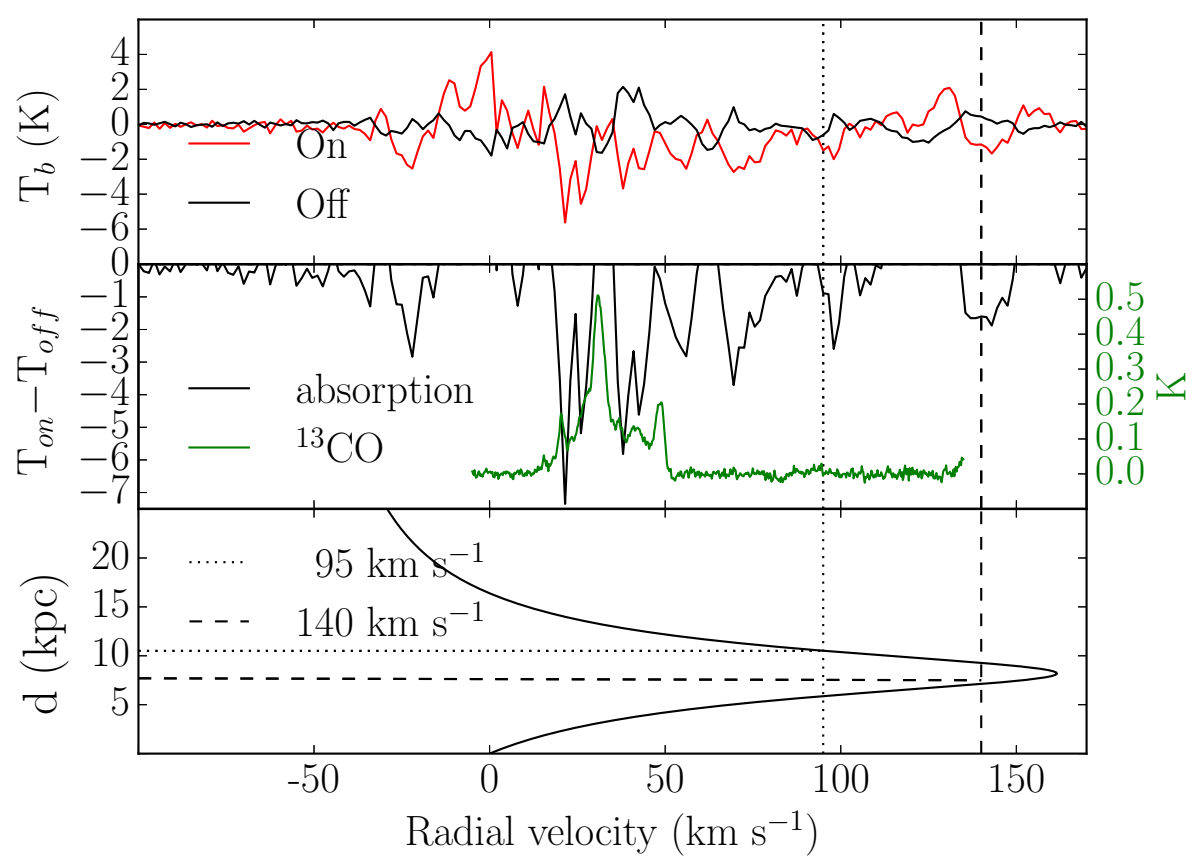

Fig. 3 The HI emission, HI absorption, and the ${ }^{13} \mathrm{CO}$ spectra of G15 from THOR and GRS data. 